\title{
Desempenho da ultra-sonografia na detecção das metástases em linfonodos axilares em mulheres com câncer de mama
}

\author{
Performance of ultrasound in the detection of axillary lymph node \\ metastases in breast cancer patients \\ Rodrigo Menezes Jales ${ }^{1}$, Emílio Francisco Marussi², Kleber Cursino de Andrade ${ }^{1}$, \\ Sophie Françoise Mauricette Derchain²
}

\section{RESUMO}

Objetivos: avaliar o desempenho de diversas características ultra-sonográficas morfológicas (12) e doplervelocimétricas (7), para detecção de metástases linfonodais em mulheres com câncer de mama. Métodos: foram incluídas 179 mulheres (181 axilas), entre janeiro e dezembro de 2004. Os exames ultra-sonográficos foram realizados com transdutor linear em tempo real (Toshiba-Power Vision-6000 (modelo SSA-370A)). Para o estudo de parâmetros morfológicos foram utilizadas freqüências entre 7,5 e $12 \mathrm{MHz}$. Para os parâmetros Doppler foi utilizada freqüência de $5 \mathrm{MHz}$. Subsequentemente, as pacientes foram submetidas a axilectomia dos níveis I, II e III (158) ou à técnica do linfonodo sentinela (23). Foram calculadas a sensibilidade, a especificidade e os valores preditivos positivo e negativo para cada parâmetro. Para a associação de parâmetros foi utilizada a árvore de decisão. Foram estabelecidos os valores de corte para as variáveis contínuas por meio da curva ROC. Resultados: ao exame ultra-sonográfico foi identificado pelo menos um linfonodo axilar em $173(96 \%)$ exames. O exame histológico detectou metástase linfonodal em 87 mulheres (48\%). As melhores sensibilidades dos critérios morfológicos foram encontradas com o volume (62\%), diâmetro ântero-posterior (62\%) e local do centro ecogênico (56\%). Embora a especificidade da invasão da gordura adjacente (100\%), regularidade das margens (92\%) e da ecotextura do parênquima (99\%) tenham sido elevadas, a sensibilidade destes parâmetros foi muito baixa. Nenhum critério dopplervelocimétrico alcançou 50\% de sensibilidade. Aárvore de decisão selecionou a localização do centro ecogênico, a ecotextura do parênquima e a regularidade das margens como melhor associação de parâmetros. Conclusão: o estadiamento dos linfonodos axilares por método não invasivo ainda é um objetivo não alcançado no tratamento das pacientes com câncer de mama.

PALAVRAS-CHAVE: Neoplasias mamárias/diagnóstico; Ultrasonografia; Linfonodos/anatomia \& histologia; Morfologia; Fluxometria por laser-doppler; Axila

\section{ABSTRACT}

Purpose: to evaluate the role of morphological (12) and Doppler velocimetry (17) ultrasonographic features, in the detection of lymph node metastases in breast cancer patients. Methods: 179 women (181 axillary cavities) were included in the study from January to December 2004. The ultrasonographic examinations were performed with a real-time linear probe (ToshibaPower Vision-6000 (model SSA-370A)). The morphological parameters were studied with a frequency of 7.5-12 MHz. A frequency of $5 \mathrm{MHz}$ was used for the Doppler velocimetry parameters. Subsequently, the women were submitted to level I, II and III axillary dissection (158), or to the sentinel lymph node technique (23). Sensitivity, specificity ${ }_{2}$ and positive and negative predictive values were calculated for each parameter. The decision tree test was used for parameter association. The cutoff points were established by the ROC curve. Results: at least one lymph node was detected in 173 (96\%) of the women by the ultrasonographic examinations. Histological examination detected lymph node metastases in 87 women

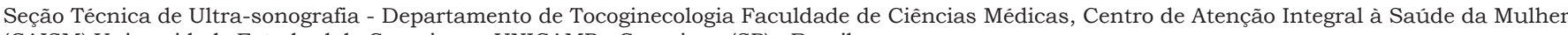
(CAISM) Universidade Estadual de Campinas - UNICAMP - Campinas (SP) - Brasil.

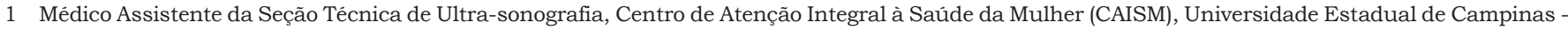
UNICAMP - Campinas (SP) - Brasil.

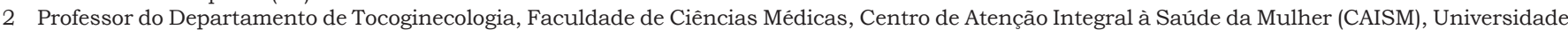
Estadual de Campinas - UNICAMP - Campinas (SP) - Brasil.

Correspondência: Rodrigo Menezes Jales

Seção Técnica de Ultra-sonografia - CAISM/UNICAMP.

Cidade Universitária Zeferino Vaz.

R Alexandre Fleming, s/ nº Campinas-SP - 13083-970 - Telefone 19- 3788-9500 - e-mail: ecografia@caism.unicamp.br 
(48\%). The best sensitivity among the morphological paramenters was found with the volume (62\%), the antero-posterior diameter $(62 \%)$ and the fatty hilum placement $(56 \%)$. Though the specificity of the extracapsular invasion (100\%), border regularity $(92 \%)$ and cortex echogenicity $(99 \%)$ were high, the sensitivity of these features was too low. None of the Doppler velocimetry parameters reached $50 \%$ sensitivity. The decision tree test selected the ultrasonographic parametners: fatty hilum placement, border regularity and cortex echogenicity, as the best parameter association. Conclusion: the detection of axillary cavity lymph node stage by a noninvasive method still remains an unfulfilled goal in the treatment of patients with breast cancer.

KEYWORDS: Breast neoplasms/diagnosis; Lymph nodes/anatomy \& histology; Morphology; Laser-doppler flowmetry; Axillary cavity

\section{Introdução}

A avaliação clínica da axila para previsão do comprometimento linfonodal histológico em mulheres com câncer da mama é falha e a dissecção axilar é necessária para o estadiamento patológico. Apesar da importância da axilectomia na avaliação prognóstica e na definição da terapia adjuvante, seu efeito no tratamento loco-regional do câncer de mama em estádios iniciais é discutivel $^{1}$. No início dos anos 90 iniciaram-se os estudos sobre a técnica do linfonodo sentinela (TLNS), o que diminuiu muito a morbidade cirúrgica e as seqüelas ${ }^{2,3}$. Menos de $25 \%$ das pacientes submetidas à TLNS apresentam sintomas relacionados à drenagem linfática, sendo a ocorrência de linfedema limitante, desprezivel ${ }^{4}$. Entretanto, pela TLNS observam-se 5 a $10 \%$ de resultados falsonegativos na avaliação de metástases axilares ${ }^{5}$.

Diversas modalidades propedêuticas de imagens têm sido estudadas na avaliação dos linfonodos axilares na tentativa de melhorar a sensibilidade e especificidade do exame clínico. Entretanto, os resultados expostos na literatura demonstram que a mamografia, a tomografia computadorizada e a tomografia por emissão de pósitrons (PET-scan) também apresentam baixo desempenho6-8. Apenas a ressonância nuclear magnética, exame de custo muito elevado, apresenta maior sensibilidade, chegando a $82 \%{ }^{9}$.

A ultra-sonografia, método propedêutico disponivel com custo relativamente baixo, tem mostrado desempenho superior à mamografia e ao exame físico na identificação de linfonodos comprometidos $^{6,10,11}$. Assim, a ultra-sonografia poderia contribuir no diagnóstico dos linfonodos axilares comprometidos por neoplasia, podendo ajudar no planejamento cirúrgico ${ }^{12}$. Sob visão ultra-sonográfica também pode ser realizada punção aspirativa por agulha fina dos linfonodos suspeitos, com boa especificidade $(96 \%)$, porém baixa sensibilidade $(57 \%)$ e muito desconforto para a paciente ${ }^{13}$.

A ultra-sonografia utiliza a emissão e detecção de ondas sonoras para geração de imagens, permitindo uma avaliação morfológica dos tecidos, e, por meio do recurso Doppler, estuda o fluxo sanguíneo ${ }^{11}$. A avaliação ultra-sonográfica dos linfonodos axilares demonstrou ampla variação na sensibilidade, que variou de 56 a $100 \%$, e na especificidade, que variou de 9,5 a 98\% ${ }^{14-17}$. Entretanto, os estudos não são uniformes quanto às combinações de características ultra-sonográficas morfológicas ou dopplervelocimétricas como critério de invasão neoplásica linfonodal. Também não especificam a seleção dos critérios utilizados para definição de invasão linfonodal. Este estudo tem como finalidade avaliar o desempenho de diversas características ultrasonográficos morfológicas e dopplervelocimétricas na detecção de linfonodos invadidos por neoplasia em mulheres com câncer de mama.

\section{Métodos}

Este é estudo de validação de teste diagnóstico. Todas as pacientes com câncer de mama (invasivo ou in situ), internadas na enfermaria de oncologia cirúrgica do Centro de Atenção Integral à Saúde da Mulher (CAISM), entre janeiro e dezembro de 2004, para mastectomia (114) ou quadrantectomia (67) com indicação de avaliação cirúrgica da axila foram convidadas a participar do estudo. Foram incluídas as mulheres com diagnóstico confirmado por biópsia de agulha grossa ou biópsia excisional com qualquer estadiamento e independentemente do exame clinico axilar. Foram excluídas as mulheres submetidas à quimioterapia ou radioterapia neoadjuvante. Foram obtidos a partir dos prontuários, a idade, o índice de massa corpórea, o quadrante mamário acometido, o tamanho patológico e o tipo histológico do tumor e a técnica de avaliação axilar. O projeto foi aprovado pelo Comitê de Ética em Pesquisa da Faculdade de Ciências Médicas da Universidade Estadual de Campinas (Unicamp), protocolado sob número 473/2003. Após assinarem o termo de consentimento livre e esclarecido, as mulheres eram submetidas à ultrasonografia axilar.

Foram estudados 12 parâmetros morfológicos e sete parâmetros dopplervelocimétricos, descritos a seguir e ilustrados nas Figuras 1 e 2: diâmetro longi- 
tudinal: maior eixo do linfonodo em $\mathrm{mm}$, sendo considerado positivo quando maior que 13,9 mm (Figuras 1-A e 2-a); diâmetro ântero-posterior: maior eixo perpendicular ao diâmetro longitudinal, em $\mathrm{mm}$, sendo considerado positivo quando maior que $6,2 \mathrm{~mm}$ (Figuras 1-A e 2-a); diâmetro transverso: maior diâmetro do linfonodo, numa secção perpendicular à utilizada para a medida do diâmetro longitudinal, em $\mathrm{mm}$, sendo considerado positivo quando maior que $8,3 \mathrm{~mm}$ (Figura 1-A e 2-a). O volume foi calculado pela multiplicação dos diâmetros longitudinal, ânteroposterior e transverso, multiplicado por 0,52 , sendo considerado positivo quando maior que $3 \mathrm{~cm}^{3}$. A forma do linfonodo foi calculada pela divisão do diâmetro longitudinal pelo transverso, categorizada em elipsóide (divisão $\geq 1,5$ (negativo)) ou arredondada (divisão $<1,5$ (positivo) $)^{18}$. O indice de Solbiati corresponde à divisão entre os diâmetros longitudinal e transverso, classificado como positivo quando menor que 2,5 . A espessura máxima do parênquima foi considerada positiva quando $\geq 2,9 \mathrm{~mm}$ (Figuras $1-\mathrm{B}$ e $2-\mathrm{b}$ ). O local do centro ecogênico foi definido como a posição do centro ecogênico em relação ao linfonodo, considerado positivo quando desviado (Figuras 1-D-2 e 2-c) ou ausente (Figuras 1-D-3 e 2-d) e negativo quando central (Figuras 1-D-1). Ecotextura do parênquima: regularidade da textura do parênquima linfonodal, categorizada em homogênea (negativo) (Figura 1-G1 e heterogênea (positivo) (Figuras 1-G-2 e 2-e); regularidade da espessura do parênquima: classificada em regular (positivo) (Figura 1-E-1 e irregular (negativo) (Figuras 1-E-2 e 2-f). As margens do linfonodo foram classificadas em irregulares (positivo) (Figuras $1-\mathrm{C}-1$ e 2 -g) e regulares (negativo) (Figuras 1-C-2 e 2-g) e a infiltração da gordura adjacente foi classificada em presente (positivo) (Figuras 1-F-2 e 2-h) ou ausente (negativo) (Figura 1-F-1). A dopplervelocimetria foi considerada suspeita: característica do linfonodo, utilizando a presença de vasos na espessura do parênquima, (detectados pelo recurso dopplervelocimetria de potência), classificada como presente (positivo) (Figuras 1-H-2 e 2-i) ou ausente (negati-

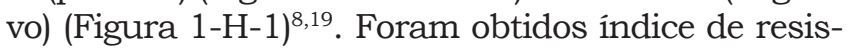
tência: grau de resistência vascular ao fluxo sanguíneo, determinada pela fórmula: (velocidade máxima - velocidade minima/velocidade máxima) (Figura 2j); indice de pulsatilidade: grau de resistência vascular ao fluxo sanguíneo determinada pela fórmula: (velocidade máxima - velocidade mínima/velocidade média) (Figura 2-j); relação velocidade máxima/velocidade minima: grau de resistência vascular ao fluxo sanguíneo determinada pela fórmula: velocidade máxima/ velocidade minima (Figura $2-\mathrm{j}$ ); velocidade máxima do fluxo arterial: classificada em positiva quando $\geq 10,8 \mathrm{~cm} / \mathrm{s}$; velocidade média do fluxo arterial: classificada em positiva quando $\geq 6,3 \mathrm{~cm} / \mathrm{s}$; velocidade minima do fluxo arterial: classificada em positiva quando $\geq 2,5 \mathrm{~cm} / \mathrm{s}$.

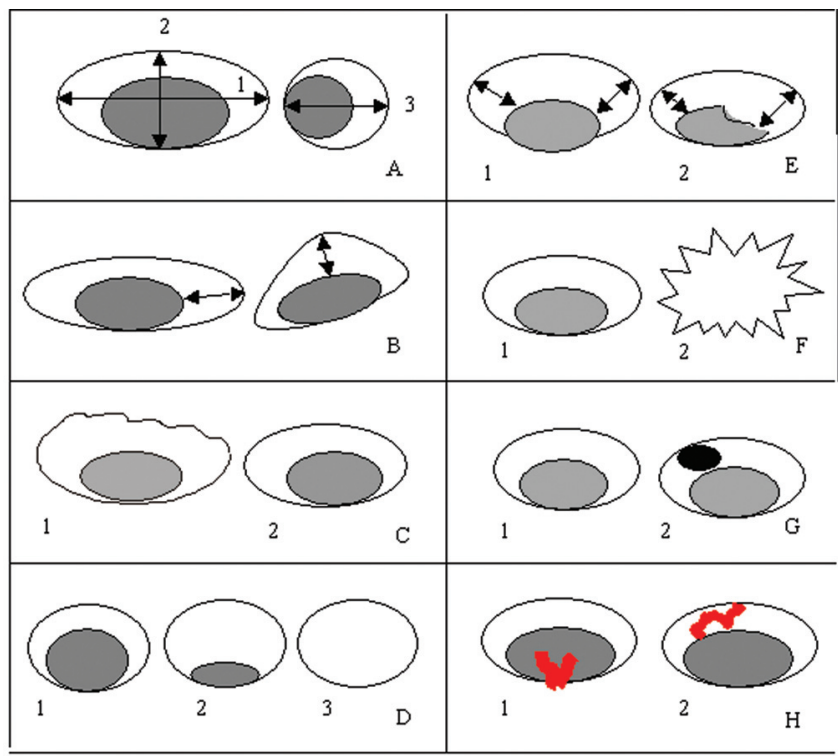

Figura 1 - Varıaveıs ultra-sonogratıcas utılızadas na avalıaçäo do acometımento Iıntonodal axilar. A) Diâmetros longitudinal (1), ântero-posterior (2) e transverso (3). B) Exemplos de medidas da espessura máxima do parênquima. C) Margens irregular (1) e regular (2). D) Centro ecogênico central (1), desviado (2) e ausente (3). E) Espessura do parênquima regular (1) e irregular (2). F) Invasão da gordura adjacente ausente (1) e presente (2). G) Ecotextura do parênquima homogênea (1) e heterogênea (2). H) Doppler suspeito negativo (1) e positivo (2).
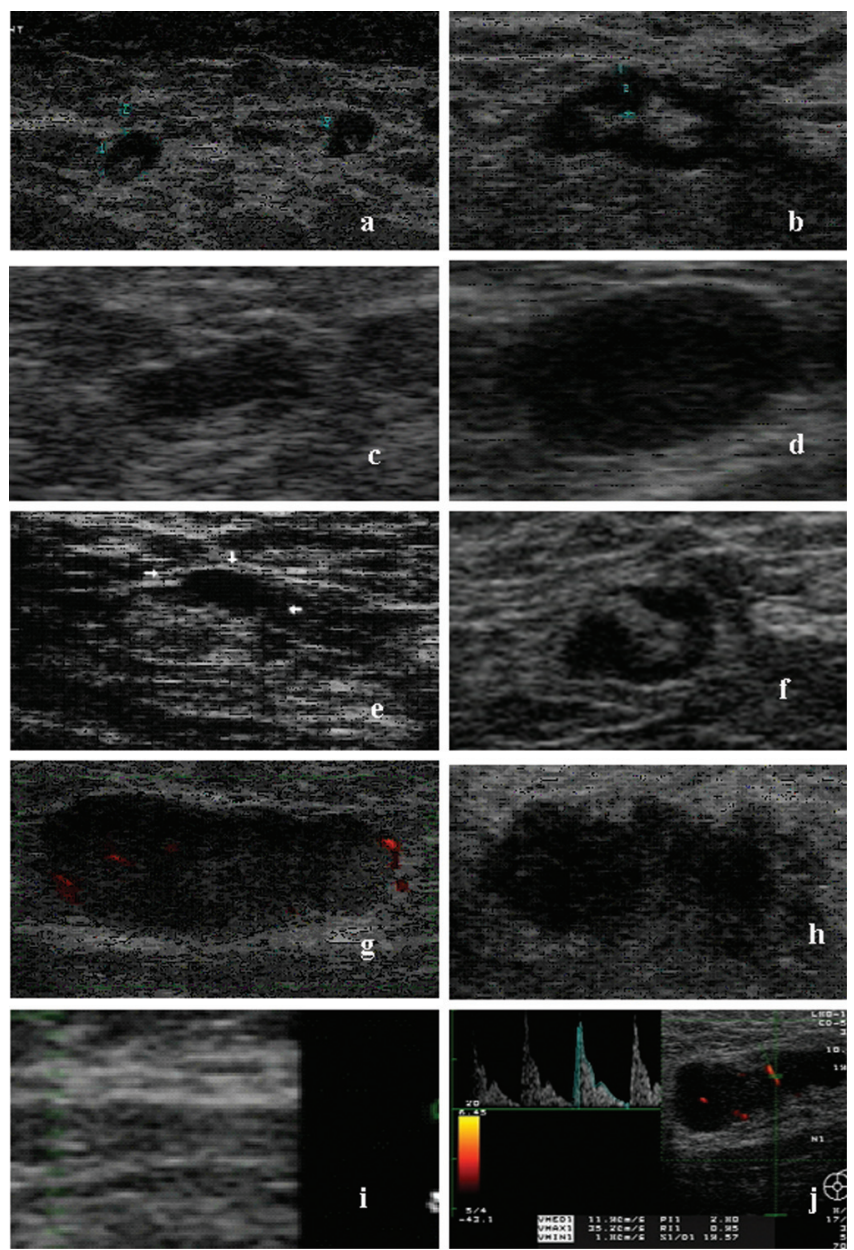

Figura 2 - Exames ultra-sonográficos na avaliação do acometimento linfonodal axilar. a) Medida dos diâmetros longitudinal (1), ântero-posterior (2) e transverso (3). b) Medida da espessura máxima do parênquima (1). c) Centro ecogênico desviado. d) centro ecogênico ausente. e) Parênquima heterogêneo (setas). f) Espessura do parênquima irregular. g) Margens irregulares. h) Invasão da gordura adjacente. i) Doppler suspeito. j) Doppler de potência e espectral (com ajuste do ângulo de insonação). 
Todos os exames foram realizados em aparelho Toshiba-Power Vision-6000 (modelo SSA-370A), equipado com transdutor linear de uso manual de $80 \mathrm{~mm}$, multifreqüencial. Para morfologia em escala de cinza foram utilizadas freqüências entre 7,5 e $12 \mathrm{MHz}$. Para os recursos dopplervelocimetria de potência e pulsado foi utilizada freqüência de 5 $\mathrm{MHz}$. Os exames foram realizados por RMJ, com a paciente em decúbito dorsal, com as mãos posicionadas atrás da cabeça. Quando detectados mais de um linfonodo, foi selecionado para análise ultra-sonográfica o que apresentava mais alterações em relação ao normal (Figura 1). Cada exame levou cerca de 10 minutos para ser realizado.

No dia seguinte as mulheres eram submetidas ao procedimento cirúrgico preconizado segundo a rotina do serviço de mastologia do CAISM. A abordagem da axila foi realizada com axilectomia dos niveis I, II e III em 158 casos, com retirada de uma média de 17,5 linfonodos (desvio padrão 8,9) e 23 mulheres foram submetidas à TLNS com retirada de uma média de 3,4 linfonodos (desvio padrão: 2,9).

$\mathrm{O}$ estudo anatomopatológico dos linfonodos axilares foi conduzido segundo as normas do Departamento de Anatomia Patológica da Faculdade de Ciências Médicas (FCM) da Unicamp. O cirurgião e o patologista não tinham conhecimento dos achados ultra-sonográficos. Os linfonodos foram classificados como positivos quando pelo menos um linfonodo apresentava invasão ou microinvasão pela neoplasia.

Para a análise estatística, inicialmente foi elaborada tabela descritiva para verificar a homogeneidade da amostra em relação às características das mulheres (idade e índice de massa corpórea), da doença (tamanho patológico do tumor, quadrante acometido, diagnóstico histológico) e da técnica de avaliação axilar em relação à invasão linfonodal. A associação entre as variáveis foi calculada através dos testes $\chi^{2}$ e exato de Fisher. A seguir foram calculados a sensibilidade, a especificidade, o valor preditivo positivo (VPP) e o valor preditivo negativo (VPN) das variáveis ultra-sonográficas isoladamente. Para as variáveis contínuas indice de Solbiati, espessura máxima do parênquima, diâmetros longitudinal, ântero-posterior e transverso, volume do linfonodo, índice de pulsatilidade, indice de resistência, velocidade máxima, média e mínima e relação velocidade máxima/mínima foi obtido o melhor valor de corte por meio da receiver operator characteristic curve (ROC). Após a análise univariada, foi realizada uma análise multivariada através do programa de árvore de decisão. A árvore foi construída para mostrar qual é a melhor associação de variáveis e como devem ser abordadas ${ }^{20}$. Toda análise estatística foi realizada por meio do programa SAS. Foi considerado um nível de significância de 95\%.

\section{Resultados}

Foram incluídas no estudo 179 mulheres, das quais duas apresentaram neoplasia em ambas as mamas, totalizando 181 axilas. Ao exame ultrasonográfico foi identificado pelo menos um linfonodo axilar em 173 (96\%) exames. Em relação à avaliação histológica, dos 23 casos submetidos à TLNS, quatro apresentavam invasão do linfonodo. Entre as 158 axilas dissecadas, 94 apresentavam invasão linfonodal em pelo menos um linfonodo. Assim, a taxa de invasão linfonodal foi de $48 \%$, sendo que em seis desses casos havia apenas micrometástases $(<2 \mathrm{~mm})$. A idade, o índice de massa corpórea e o quadrante acometido não estiveram significativamente relacionados com o comprometimento linfonodal axilar. Já o maior tamanho do tumor, o diagnóstico histológico invasor e o esvaziamento axilar completo estiveram significativamente relacionados com maior comprometimento linfonodal (Tabela 1).

Tabela 1 - Características das mulheres incluídas no estudo segundo o comprometimento axilar.

\begin{tabular}{|c|c|c|c|}
\hline $\begin{array}{l}\text { Características } \\
\text { da paciente }\end{array}$ & $\begin{array}{c}\text { Linfonodos } \\
\text { livres } \\
n(\%)\end{array}$ & $\begin{array}{c}\text { Linfonodo(s) } \\
\text { comprometido(s) } \\
\text { n (\%) }\end{array}$ & ) \\
\hline Idade & & & $0,1868^{*}$ \\
\hline$<50$ anos & $29(31 \%)$ & $36(41 \%)$ & \\
\hline$\geq 50$ anos & $65(69 \%)$ & $51(59 \%)$ & \\
\hline IMC & & & $0,7112^{*}$ \\
\hline Normal & $68(72 \%)$ & $66(76 \%)$ & \\
\hline Sobrepeso >30 & $26(28 \%)$ & $21(24 \%)$ & \\
\hline Tamanho patológico do tumor & & & $0,009 \dagger$ \\
\hline$\leq 2 \mathrm{~cm}$ & $54(60 \%)$ & $32(38 \%)$ & \\
\hline$>2 \leq 5 \mathrm{~cm}$ & $34(38 \%)$ & $48(56 \%)$ & \\
\hline$>5 \mathrm{~cm}$ & $2(2 \%)$ & $5(6 \%)$ & \\
\hline Quadrante & & & $0,37^{*}$ \\
\hline mediais & $43(46 \%)$ & $34(39 \%)$ & \\
\hline laterais & $50(54 \%)$ & $53(61 \%)$ & \\
\hline Diagnóstico histológico & & & $<0,0001 \dagger$ \\
\hline Ductal in situ & $9(10 \%)$ & $0(0 \%)$ & \\
\hline Ductal invasor & $65(69 \%)$ & $82(94 \%)$ & \\
\hline Outro invasor & $19(20 \%)$ & $5(6 \%)$ & \\
\hline Técnica de avaliação axilar & & & $0,00341 \dagger$ \\
\hline Linfonodo sentinela & $19(20 \%)$ & $4(5 \%)$ & \\
\hline Axilectomia radical & $75(80 \%)$ & $83(95 \%)$ & \\
\hline IMC: Índice de massa corpórea & *: teste do $\chi^{2}$ & $t$ : teste exato de Fishe & \\
\hline
\end{tabular}


Tabela 2 - Desempenho, em ordem crescente de sensibilidade, das características morfológicas e do Doppler na detecção do comprometimento linfonodal.

\begin{tabular}{|c|c|c|c|c|}
\hline \multirow{2}{*}{$\frac{\text { Características }}{\text { Morfológicas }}$} & \multicolumn{3}{|c|}{ Sensibilidade Especificidade VPP } & \multirow[t]{2}{*}{ VPN } \\
\hline & & & & \\
\hline Invasão da gordura adjacente & $7 \%$ & $100 \%$ & $100 \%$ & $58 \%$ \\
\hline Índice de Solbiati & $19 \%$ & $69 \%$ & $37 \%$ & $48 \%$ \\
\hline Forma & $21 \%$ & $92 \%$ & $72 \%$ & $56 \%$ \\
\hline Ecotextura do parênquima & $23 \%$ & $99 \%$ & $95 \%$ & $58 \%$ \\
\hline $\begin{array}{l}\text { Regularidade da espessura do } \\
\text { parênquima }\end{array}$ & $26 \%$ & $92 \%$ & $77 \%$ & $58 \%$ \\
\hline Regularidade das margens & $34 \%$ & $96 \%$ & $88 \%$ & $61 \%$ \\
\hline $\begin{array}{l}\text { Espessura máxima do } \\
\text { parênquima }\end{array}$ & $41 \%$ & $82 \%$ & $68 \%$ & $60 \%$ \\
\hline Diâmetro longitudinal & $52 \%$ & $56 \%$ & $52 \%$ & $56 \%$ \\
\hline Diâmetro transverso & $53 \%$ & $64 \%$ & $57 \%$ & $59 \%$ \\
\hline Local do centro ecogênico & $56 \%$ & $89 \%$ & $83 \%$ & $69 \%$ \\
\hline Diâmetro ântero-posterior & $62 \%$ & $56 \%$ & $57 \%$ & $62 \%$ \\
\hline Volume & $62 \%$ & $55 \%$ & $56 \%$ & $61 \%$ \\
\hline \multicolumn{5}{|l|}{ Doppler } \\
\hline Índice de pulsatilidade & $41 \%$ & $74 \%$ & $60 \%$ & $58 \%$ \\
\hline Relação sístole/diástole & $42 \%$ & $74 \%$ & $60 \%$ & $58 \%$ \\
\hline Velocidade média & $45 \%$ & $82 \%$ & $70 \%$ & $62 \%$ \\
\hline Índice de resistência & $46 \%$ & $73 \%$ & $61 \%$ & $59 \%$ \\
\hline Velocidade máxima & $47 \%$ & $84 \%$ & $73 \%$ & $63 \%$ \\
\hline Doppler suspeito & $47 \%$ & $85 \%$ & $74 \%$ & $63 \%$ \\
\hline Velocidade mínima & $48 \%$ & $75 \%$ & $65 \%$ & $61 \%$ \\
\hline
\end{tabular}

Valores de corte calculados pela curva ROC utilizados para as variáveis: índice de Solbiati $=2,5$, espessura máxima do parênquima $=2,9 \mathrm{~mm}$, diâmetro longitudinal $=13,9$ $\mathrm{mm}$, diâmetro ântero-posterior $=6,2 \mathrm{~mm}$, diâmetro transverso $=8,3 \mathrm{~mm}$, volume do linfonodo

$=3 \mathrm{~cm}^{3}$, índice de pulsatilidade $=1,1$, índice de resistência $=0,7$, velocidade máxima $=$ $10,8 \mathrm{~cm} / \mathrm{s}$, velocidade média $=6,3 \mathrm{~cm} / \mathrm{s}$, velocidade mínima $=2,5 \mathrm{~cm} / \mathrm{s}$ e relação velocidade máxima/mínima $=3,1$ VPP $=$ valor preditivo positivo, $\mathrm{VPN}=$ valor preditivo negativo .
Na Tabela 2 podemos observar que nenhum critério morfológico isoladamente apresentou sensibilidade alta. As melhores sensibilidades foram associadas ao volume do linfonodo (62\%), diâmetro ântero-posterior (62\%) e local do centro ecogênico (56\%). Embora a especificidade da invasão da gordura adjacente (100\%), da ecotextura do parênquima (99\%) e da regularidade das margens $(96 \%)$ tenha sido muito elevada, a sensibilidade destes parâmetros foi muito baixa. Nenhum critério dopplervelocimétrico alcançou $50 \%$ de sensibilidade.

Por meio da árvore de decisão pode-se observar que a primeira variável a ser selecionada foi o local do centro ecogênico. O centro ecogênico estava ausente ou desviado em 49 das 87 axilas comprometidas e apresentou um VPP de $83 \%$. Para os casos em que o centro ecogênico estava posicionado na região central, a árvore de decisão selecionou a ecotextura do parênquima como variável importante. Esta foi considerada heterogênea em 10 casos, todos de axila comprometida. Quando a ecotextura foi homogênea, o último parâmetro selecionado foi a regularidade das bordas. Nos seis casos em que a borda era irregular, quatro apresentavam axila positiva (VPP $=67 \%$ ). Entretanto, a sensibilidade global da arvore de decisão foi de apenas $72 \%$, com especificidade de $87 \%$, VPP de $84 \%$ e VPN de $77 \%$ (Tabela 3 ).

Tabela 3 - Árvore de decisão com os aspectos ultra-sonográficos mais relacionados com o acometimento linfonodal axilar

Características selecionadas pela metodologia de àrvore de decisão (CHAID)*

Resultados

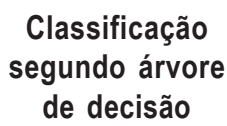

Local do centro ecogênico Ecotextura do parênquima Regularidade da imagem $(p<0,0001)$ $(p<0,0001)$

Ausente ou

Desviado

Central

Heterogêneo

Homogêneo

Total

Total

Matriz de classificação

Classificação
segundo árvore

de decisão

Pãa-ouro

Positivo Negativo Total

$\begin{array}{lll}63 & 12 \quad 75\end{array}$

$\begin{array}{clll}\text { Negativo } & 24 & 82 & 106 \\ \text { Total } & 87 & 94 & 181\end{array}$

$(p=0,0462)$

\begin{tabular}{ccrrrr} 
Positivo & Negativo & \multicolumn{2}{c}{ Positivo } & \multicolumn{2}{c}{ Negativo } \\
n & n & n & \multicolumn{1}{c}{$\%$} & n & $\%$ \\
\hline 59 & 0 & 49 & $83 \%$ & 10 & 17 \\
10 & 0 & 10 & $100 \%$ & 0 & 0 \\
6 & 0 & 4 & $67 \%$ & 2 & 33 \\
0 & 106 & 24 & $23 \%$ & 82 & 77 \\
6 & 106 & 28 & $25 \%$ & 84 & 75 \\
16 & 106 & 38 & $31 \%$ & 84 & 69 \\
75 & 106 & 87 & & 94 &
\end{tabular}

Irregular

Regular

Total 


\section{Discussão}

Neste estudo observamos que nenhum critério ultra-sonográfico morfológico ou dopplervelocimétrico apresentou sensibilidade adequada quando avaliado isoladamente. Quando analisados por meio de árvore de decisão, a seqüência sugerida para avaliação dos critérios que possam definir o estado da axila foi o local do centro ecogênico, a ecotextura do parênquima e a regularidade das margens, aumentando a sensibilidade.

O local do centro ecogênico é freqüentemente utilizado na avaliação linfonodal ${ }^{10,21}$. O centro ecogênico corresponde à região central do linfonodo onde é coletada a linfa. Quando a célula neoplásica penetra no linfonodo pelos vasos linfáticos na periferia do parênquima e se multiplica no parênquima, o centro é deslocado. Essa alteração é facilmente identificável à ultra-sonografia. Entretanto, este processo de deslocamento do centro ecogênico é tardio, e só ocorre quando a invasão linfonodal já é macroscópica, tendo baixa sensibilidade nos casos iniciais. O deslocamento do centro ecogênico também ocorre nos processos inflamatórios ${ }^{21,22}$.

A ecotextura do parênquima e a regularidade da margem precisam ser avaliadas em alta resolução, obtida com transdutores modernos de alta freqüência, sendo citados mais recentemente na literatura ${ }^{14,23}$. Aparentemente, tratam-se de parâmetros promissores na avaliação linfonodal pela ultra-sonografia. Da mesma forma que o centro ecogênico, a ecotextura e as margens do linfonodo alteraram-se diante da multiplicação das células neoplásicas no linfonodo.

Embora as alterações ultra-sonográficas sejam mais facilmente identificáveis na doença linfonodal avançada, dos seis casos com micrometástase axilar, dois apresentaram ausência do centro ecogênico e um apresentou irregularidade da margem à ultra-sonografia.

Foi identificado pelo menos um linfonodo à ultra-sonografia na grande maioria dos casos. Esta taxa de detecção é superior àquela encontrada em estudos menos recentes $(7 \text { a } 65 \%)^{10}$. A melhora na taxa de detecção de linfonodos pela ultrasonografia nos estudos mais recentes se deve, provavelmente, à utilização de equipamentos com melhor definição. É importante destacar que não houve diferença na identificação de linfonodos nas mulheres com e sem invasão histológica da axila. Relatos recentes na literatura demonstram que atualmente a dificuldade não está em identificar os linfonodos, mas sim em diferenciar aqueles acometidos pela neoplasia dos normais ou reacio- nais ${ }^{24}$. O desempenho da ultra-sonografia no presente estudo foi semelhante ao obtido in vitro ${ }^{25}$. Há pouco mais de uma década, os aparelhos disponiveis identificavam, predominantemente, os linfonodos comprometidos, pois os linfonodos normais não eram identificados na gordura axilar adjacente $^{6}$. Utilizando-se transdutores de 3,5 $\mathrm{MHz}$, era considerado comprometido pela neoplasia qualquer linfonodo identificado pelo ultra-som. Apenas os linfonodos em estados avançados de invasão neoplásica eram visiveis ${ }^{15}$. Atualmente, com a utilização de sondas com até $12 \mathrm{MHz}$, é mais fácil diferenciar os linfonodos da gordura adjacente, entretanto ainda não se consegue diferenciar com segurança os linfonodos inflamatórios dos neoplá$\operatorname{sicos}^{24}$.

A ultra-sonografia não apresentou uma sensibilidade suficiente para ser utilizada isoladamente na avaliação dos linfonodos axilares. Entretanto, o estudo ultra-sonográfico pode complementar o exame físico na indicação da TLNS. A associação da ultra-sonografia com a TLNS obtém melhores resultados do que a TLNS isoladamen$t^{26}$. Optou-se, neste estudo, por incluir os casos submetidos a TLNS: aceitando-se uma taxa de falso-negativos de até $10 \%$ pelo linfonodo sentinela, teríamos, no máximo, dois casos classificados inadequadamente.

Uma das grandes limitações dos estudos de imagem para a avaliação da axila está relacionada à relação entre o linfonodo examinado e o linfonodo comprometido histologicamente. Nos casos com metástase linfonodal, não há como ter certeza de que o linfonodo avaliado pela ultrasonografia corresponde ao linfonodo histologicamente comprometido. Este problema, comum a todos os estudos, poderia ser minimizado com a punção do linfonodo identificado pelo ultra-som. Mas a citologia também apresenta casos falsos positivos e negativos ${ }^{13}$. Outra opção ética é o estudo ultra-sonográfico in vitro. Entretanto, essa técnica altera as condições em que os linfonodos seriam avaliados na prática clínica e impossibilita o estudo dopplervelocimétrico ${ }^{18}$.

Assim, ao realizar exame ultra-sonográfico da axila, podemos apenas supor que, identificando-se um linfonodo com centro ecogênico desviado, alteração da textura do parênquima e margens irregulares à ultra-sonografia, é muito provável que se trate de linfonodo acometido pela neoplasia.

Concluímos que no presente estudo, a ultrasonografia convencional da axila com até $12 \mathrm{MHz}$ complementada pela dopplervelocimetria não deve ser utilizada como única ferramenta propedêutica na seleção de quais pacientes se beneficiaram da axilectomia. Entretanto, talvez a ultra-sonografia 
possa contribuir para a detecção de linfonodos no nível três da axila (não palpável). Além disso, nos casos mais avançados de invasão linfonodal, a TLNS pode não identificar metástases pela obstrução da rede linfática ${ }^{26}$. Nestes casos o ultra-som poderia auxiliar o cirurgião na retirada deste linfonodo.

\section{Referências}

1. Kingsmore DB, Hole DJ, Gillis CR, George WD. Axillary recurrence in breast cancer. Eur J Surg Oncol. 2005;31(3):226-31.

2. Sener SF, Winchester DJ, Martz CH, Feldman JL, Cavanaugh JA, Winchester DP, et al. Lymphedema after sentinel lymphadenectomy for breast carcinoma. Cancer. 2001;92(4):748-52.

3. Rönka RH, Pamilo MS, Von Smitten KA, Leidenius $\mathrm{MH}$. Breast lymphedema after breast conserving treatment. Acta Oncol. 2004;43(6):551-7.

4. Rönkä RH, Von Smitten KA, Tasmuth T, Leidenius M. One-year morbidity after sentinel node biopsy and breast surgery. Breast. 2005;14(1):28-36.

5. Barranger E, Grahek D, Antoine M, Talbot JN, Uzan S. Sentinel lymph node biopsy in breast cancer: technical aspects and results. Ann Chir. 2003;128(3):144-9.

6. Pamilo M, Soiva M, Lavast EM. Real-time ultrasound, axillary mammography, and clinical examination in the detection of axillary lymph node metastases in breast cancer patients. J Ultrasound Med. 1989;8(3):115-20.

7. Yuen S, Yamada K, Goto M, Sawai K, Nishimura T. CT-based evaluation of axillary sentinel lymph node status in breast cancer: value of added contrastenhanced study. Acta Radiol. 2004;45(7):730-7.

8. Wahl RL, Siegel BA, Coleman RE, Gatsonis CG; PET Study Group. Prospective multicenter study of axillary nodal staging by positron emission tomography in breast cancer: a report of the staging breast cancer with PET Study Group. J Clin Oncol. 2004;22(2):277-85.

9. Michel SC, Keller TM, Frohlich JM, Fink D, Caduff $\mathrm{R}$, Seifert B, et al. Preoperative breast cancer staging: MR imaging of the axilla with ultrasmall superparamagnetic iron oxide enhancement. Radiology. 2002;225(2):527-36.

10. Yang WT, Ahuja A, Tang A, Suen M, King W, Metreweli C. High resolution sonographic detection of axillary lymph node metastases in breast cancer. J Ultrasound Med. 1996;15(3):241-6.

11. Stavros TA. Evaluation of regional lymph nodes in breast cancer patients. In: Donnellan K, editor. Breast ultrasound. Philadelphia: Lippincott Williams \& Wilkins; 2004. p. 834-76.

12. Strauss HG, Lampe D, Methfessel G, Buchmann J. Preoperative axilla sonography in breast tumor suspected of malignancy: a diagnostic advantage? Ultraschall Med. 1998;19(2):70-7.
13. Kuenen-Boumeester V, Menke-Pluymers M, de Kanter AY, Obdeijn IM, Urich D, Van Der Kwast TH. Ultrasound-guided fine needle aspiration cytology of axillary lymph nodes in breast cancer patients. A preoperative staging procedure. Eur J Cancer. 2003;39(2):170-4.

14. Shetty MK, Carpenter WS. Sonographic evaluation of isolated abnormal axillary lymph nodes identified on mammograms. J Ultrasound Med. 2004;23(1):6371.

15. de Freitas R Jr, Costa MV, Schneider SV, Nicolau MA, Marussi E. Accuracy of ultrasound and clinical examination in the diagnosis of axillary lymph node metastases in breast cancer. Eur J Surg Oncol. 1991;17(3):240-4.

16. Yang WT, Metreweli C. Colour Doppler flow in normal axillary lymph nodes. Br J Radiol. 1998;71(844):381-3.

17. Walsh JS, Dixon JM, Chetty U, Paterson D. Colour Doppler studies of axillary node metastases in breast carcinoma. Clin Radiol. 1994;49(3):189-91.

18. Feu F, Tresserra F, Fabregas R, Navarro B, Grases PJ, Suris JC, et al. Metastatic breast carcinoma in axillary lymph nodes: in vitro US detection. Radiology. 1997;205(3):831-5.

19. Yang WT, Chang J, Metrewelli C. Patients with breast cancer: differences in color Doppler flow and gray-scale US features of benign and malignant axillary lymph nodes. Radiology. 2000;215(2):56873.

20.Brieman L, Friedman JH, Olshen RA, Stone CJ. Classification and regression trees. Pacific Grove: Wadworth \& Brooks/Cole Advanced Books; 1984.

21.Yang WT, Ahuja A, Tang A, Suen M, King W, Metreweli C. Ultrasonographic demonstration of normal axillary lymph nodes: a learning curve. J Ultrasound Med. 1995;14(11):823-7.

22. Verbanck J, Vandewiele I, De Winter H, Tytgat J, Van Aelst F, Tanghe W. Value of axillary ultrasonography and sonographically guided puncture of axillary nodes: a prospective study in 144 consecutive patients. J Clin Ultrasound. 1997;25(2):53-6.

23. Rajesh YS, Ellenbogen S, Banerjee B. Preoperative axillary ultrasound scan: its accuracy in assessing the axillary nodal status in carcinoma breast. Breast. 2002;11(1):49-52.

24.Zornoza A, Pina L, Sola J, Martinez-Regueira F, Zornoza G. Imaging techniques for axillary lymph node staging in breast cancer. Rev Med Univ Navarra. 2004;48(3):9-13.

25. Tateishi T, Machi J, Feleppa E, Oishi R, Furumoto $\mathrm{N}$, McCarthy LJ, et al. In vitro B-mode ultrasonographic criteria for diagnosing axillary lymph node metastasis of breast cancer. J Ultrasound Med. 1999;18(5):349-56.

26. Sato K, Tamaki K, Tsuda H, Kosuda S, Kusano S, Hiraide $\mathrm{H}$, et al. Utility of axillary ultrasound examination to select breast cancer patients suited for optimal sentinel node biopsy. Am J Surg. 2004;187(6):679-83. 